\title{
A Remote Flipped Classroom Approach to Teaching Introductory Biomedical Engineering During COVID-19
}

\author{
Kaitlin C. Fogg (1) ${ }^{1}$ and Samantha J. Maki ${ }^{2,3}$ \\ ${ }^{1}$ School of Chemical, Biological, and Environmental Engineering, Oregon State University, Corvallis, OR 97331, USA; \\ ${ }^{2}$ Department of Educational Leadership and Policy, University of Wisconsin - Oshkosh, Oshkosh, WI 54901, USA; and ${ }^{3}$ Tribal \\ Education, Lac Du Flambeau Band of Lake Superior Chippewa Indians, Lac du Flambeau, WI 54538, USA
}

(Received 20 June 2020; accepted 17 July 2020; published online 10 August 2020)

\section{CHALLENGE STATEMENT}

At the institution of implementation, terms are divided into 10-week sessions. Due to COVID-19, the university decided to transition all courses to remote delivery two weeks before the beginning of the Spring Quarter. This enabled restructuring the courses taught in the Spring Quarter for remote delivery before the start of the class. This rapid shift to online learning presented unique challenges related to the specific class; however, many of the approaches to mediating these challenges could be applied broadly across biomedical engineering curricula.

Biomedical Engineering Principles is a required course taught to Juniors in Bioengineering. The course learning objectives are the following:

1. Knowledge of contemporary issues in biomedical engineering.

2. General knowledge of and ability to apply physiology.

3. An ability to apply advanced mathematics, science, and engineering to solve problems at the interface of engineering and biology, including those associated with the interaction between living and nonliving materials and systems.

4. An ability to design experiments, make measurements on, and interpret data from living systems.

The original course design consisted of five modules (1. pulmonary physiology, 2. cardiovascular physiology, 3. biostatistics, 4. renal physiology, and 5. phar-

Address correspondence to Kaitlin C. Fogg, School of Chemical, Biological, and Environmental Engineering, Oregon State University, Corvallis, OR 97331, USA. Electronic mail: kaitlin.fogg@ oregonstate.edu macokinetics), and three group projects (1. build a MATLAB model of an artificial lung, 2. design, execute, and analyze an experiment using biosensors, 3 . give an in-class presentation on a proposed improvement to a medical device or intervention). Traditionally, homework and group projects were completed outside of class, and scheduled class time was used for delivering lectures and having students do hands-on activities and give in-class presentations. Additionally, participation was evaluated by students attending class and completing in-class activities.

The challenges for transitioning this class to a remote delivery format were the following:

1. Engaging students with the lecture content: Attending a class in person is a very different learning environment than logging in to watch a lecture online. In order to engage students with the lecture content additional measures needed to be taken to present the information in an informative and interactive way without being able to interact with the students in person.

2. Replacing the hands-on learning activities: As the students were no longer able to come into the classroom and actually make measurements on living systems a virtual experiment needed to be created that met learning objective \#4.

3. Creating a sense of community within the class: This class is the first class that the BIOE undergraduates take with only other BIOE students and has traditionally helped foster a sense of comradery and belonging to the broader BIOE community on campus. Additionally, the majority of students were removed from their daily communities and support networks. Since students were no longer able to interact with each other in 
person, additional time needed to be built into the course structure to facilitate peer to peer interactions.

4. Fostering teamwork within group projects: Traditionally, students developed a sense of cohesiveness through working on their group projects in person outside of class. Since that was no longer an option, extra scaffolding needed to be provided in order to encourage students to work together.

5. Ensuring accessibility to all students: The majority of students left campus, encountering a wide range of home and learning environments. In order to ensure this course was accessible to students with limited internet access, care giving responsibilities, and/or time restrictions, extra flexibility needed to be built into the course.

\section{NOVEL INITIATIVE}

The overall approach to transitioning to remote delivery was to integrate a flipped classroom course design with remote delivery pedagogy. Students observed recorded lectures before class and in-class lecture time was reserved for supported application of the course material. Assessments of learning were diverse, providing opportunities to evaluate comprehension of the material, application of the concepts, and deep learning and application of the course content in a team setting (Table 1).

\section{Lectures}

Instead of delivering lectures over Zoom during our scheduled class time, the lectures for the module were recorded and posted in Canvas for students to access at least one week before that module was covered in class. This ensured that students could engage with the lecture content at their own pace. Lecture slides were made in Microsoft PowerPoint and uploaded as handouts with two slides per page, ensuring the slides were numbered for later discussion in class. The lecture slides were very detailed, such that students could get all of the information necessary for comprehending the material from reading the slides alone. Lectures were recorded in 5-10 min clips, with clear indications in the lecture slides as to which slides went with which narration. A slide saying "pause" was at the end of each recorded video, making it easier to record and also easier for the students to follow along. These short videos were clearly labeled by section headings, uploaded along with the lecture handouts, and the section headings were listed under the link to the handout. This made it easy for the students to locate which section of which video held the answers for the homework or quiz associated with that module.

Students could ask questions on the lecture material at the beginning of class. Application of the lecture material was assessed using homework problems, comprehension of the lecture material was assessed using quizzes, and engagement with the lecture material was assessed using participation assignments. Ta-

TABLE 1. Summary of the course components, their original format, and the updated format for remote delivery.

\section{Course}

component

Original format

Updated format for COVID-19 remote delivery

\section{Lectures In person delivery of lectures during scheduled class time \\ Homework Done independently outside of class}

Quizzes Closed notes timed quizzes consisting of 4-5 long form word problems taken during scheduled class time. Used to assess student's comprehension of the lecture material from the previous week. Quiz grades were final Participation Participation was evaluated on student attendance during
scheduled class time

$\begin{aligned} & \text { Group pro- } \\ & \text { jects }\end{aligned}$
$\begin{aligned} & \text { Introduced in class where students had the opportunity to do } \\ & \text { relevant hands-on activities. Group projects were then } \\ & \text { worked on outside of class }\end{aligned}$

Lectures were pre-recorded and posted in 10-min intervals along with the accompanying lecture slides as a handout

Done in virtual breakout rooms of 4-5 students during scheduled class time, after which the solutions were presented

Untimed quizzes consisting of 25 short multiple choice, matching, or bundled true/false questions. Quizzes were used to assess student comprehension of lecture material from the current module, and students could go back and engage with lecture videos or handouts while taking the quiz. Each quiz could be taken twice

Participation was evaluated by students completing three activities for each module: uploading a screen shot of a tweet or retweet of an article relevant to the current module, uploading a picture of at least one page of handwritten notes, and choosing between either writing a 250 word summary or posting a one minute video that summarized an element of the current module

Worked on group projects in breakout rooms during scheduled class time. Time was spent either in manager meetings with the instructor or as a group with the instructor available for questions 
ken together these course components addressed course learning objective \#2: general knowledge and ability to apply physiology. Additionally, they collectively addressed the challenge of engaging students with the lecture content.

\section{Homework}

Instead of homework being done independently outside of class, virtual lecture time was used to work on the homework together in a discussion format and then homework was turned in as individual assignments. Homework assignments helped students meet course learning objective \#3: an ability to apply advanced mathematics, science, and engineering to solve problems at the interface of engineering and biology, including those associated with the interaction between living and nonliving materials and systems. Homework assignments consisted of long form word problems that required students to apply the mathematical concepts from the lecture notes as well as their prior knowledge of engineering concepts such as mass and energy balances.

Homework problems were posted online as well as broadcast via screen sharing, and the homework questions were read aloud. Students were then placed into breakout rooms of 4-5 students, where they would work on the problem for anywhere from 5-20 min, depending on the length and difficulty of the problem. This helped address the challenge of creating a sense of community within the class. Students could hit the "I have a question" button and receive assistance from the professor as needed. Students would receive a oneminute warning before the breakout rooms were closed, then the breakout rooms would be closed, and everyone would return to the main room. The reaction feature in Zoom was used to assess whether or not students completed the problem, and the chat feature was used to ask students if they wanted to share their answer with the class. Homework solutions were then worked through line by line, with students asking questions in the chat feature. Students then had to work out the problems themselves, either on paper or in Microsoft Word, and turn them in the following week. Lectures were recorded and edited such that the time in the breakout rooms was removed from the final recording.

\section{Quizzes}

Previously, quizzes were taken in class and used to assess comprehension of the lecture material from the previous week. Additionally, in previous iterations of this class, quizzes were mostly long form word problems. For remote delivery of a flipped classroom, the quizzes were used to assess student comprehension of the overall concepts within the lectures, whereas the homework was used to assess whether students could apply the concepts to solve mathematical problems. Quiz questions were a combination of multiple choice, matching, or bundled true/false questions. Quizzes were graded automatically in Canvas, were not timed, and could each be taken twice, with students being told which questions they got wrong the first time. Answers were shuffled every time so that students had to read through all the options to pick the correct answer.

\section{Participation}

Due to concern that students or their family members may fall ill throughout the course, attendance in the Zoom lecture was not mandatory. However, in order to assess and encourage student engagement with the material beyond the quizzes and homework assignments, each module had three required participation activities, each accounting for $1 \%$ of their overall grade. All participation assignments were graded on completion, not content. This provided a low stakes assignment to engage with the material and earn points to boost their grade.

1. Twitter: inspired by Randi Ulbricht's twitter assignment for her class (Biomedical Sciences Department, Missouri State University), students were asked to tweet or retweet an article relevant to the current module with the hashtag \#BIOE340. They could create a dummy account just for this class, and they needed to take a screenshot of the tweet and upload it to Canvas. The overall goal of this assignment was for students to gain awareness of current science news, academic research, and discussions relevant to the topics in each module. Additionally, engagement with Science Twitter allowed students to see that the concepts they were learning in class enabled them to engage with and understand research that is currently being conducted. This assignment helped students meet course learning objective \#1: knowledge of contemporary issues in biomedical engineering.

2. Notes: students were asked to write a minimum of one sheet of notes, take a picture of it, and upload it to Canvas.

3. Summary: students were asked to write a 250word summary or a one-minute video summarizing one aspect of the current module.

\section{Group Projects}

The three group projects are where students must apply their knowledge of mathematics and physiology 
to come up with creative solutions to real-world problems. Previously, group projects were introduced in class after students had the opportunity to use various biosensors in class. However, since that was no longer an option, much more detailed information needed to be provided to students beforehand. For all of the group projects, a very detailed grading rubric was provided and was discussed during lecture. Nearly half of the class time was dedicated to working on the group projects and Zoom breakout rooms were preassigned to be their project groups. Additionally, for each group project one day was devoted to "manager meetings," where each group had to fill out a form before class started stating 1 . their overall project summary, 2. their recent accomplishments, 3. current challenges, and 4. identify and commit to next steps. Both scaffolded and unscaffolded time was provided for each group project-scaffolded being manager meetings, unscaffolded being group work in breakout rooms. These in class group project sessions were provided to help address the challenge of fostering teamwork within group projects.

There were specific challenges associated with transitioning each of the three group projects to a remote learning format. For the artificial lung project, since they no longer could interact with a spirometer, a video of how spirometry worked was provided as well as the MATLAB code that the class would have normally been able to write together in class. Since observing individual computer screens would be difficult, the focus of the project was changed from being very MATLAB heavy to being more focused in creative solutions and communicating their designs effectively.

The design and analysis of an experiment using biosensors was the most difficult group project to convert to an online learning environment, since under normal circumstances the students play with the sensors for a class period, decide which sensor they wanted to learn more about, and use it to conduct an experiment on their fellow classmates. Since the students were no longer bound by the biosensors owned by the department, students were encouraged to design an experiment using any commercially available biosensor. Additionally, the virtual aspect was leveraged as an opportunity to teach students more about sample size calculation and power analysis (since they were no longer limited by how many of their classmates, they could convince to join their study). Since they could not actually run the experiment they designed, the focus of the report shifted to generating a hypothesis, designing an experiment to test the hypothesis, presentation of the data, and choosing the correct statistical test. For data "collection," students were asked to use a website that generates data that follow a Gaussian distribution given a mean and standard deviation (https://www.random.org/gaussian -distributions/). Furthermore, to generate mock data they needed to look up what average values and standard deviations of the metric of interest would be for a control group, as well as what the raw data from the biosensor of interest would look like and how those data would need to be processed in order to get the values reported. This group project helped students meet course learning objective \#4: an ability to design experiments, make measurements on, and interpret data from living systems. Additionally, changes to this group project helped address the challenge of replacing the hands-on learning activities.

The last group project was to give an in-class presentation on a proposed improvement to a medical device or intervention. The prompt for this project stayed the same, but the delivery mode changed. The challenge for this project was replacing the in-class PowerPoint presentation and receiving peer feedback. To meet these challenges, students were asked to upload a 10-12 min video that could be any format as long as it was professional. Suggested formats: a PowerPoint Presentation with voice over (similar to the recorded lectures), a video with props pretending you're giving a demonstration of your product, a music video, a sales pitch video to investors, an animation detailing your device, etc. In-class peer review was replaced by a "gallery assessment" assignment, where students needed to watch two videos from other groups and provide constructive feedback. In order to ensure it was constructive feedback and not unhelpful criticism, students were provided with a handout detailing how to give constructive feedback. Similar to the participation assignments, the gallery assessment was graded on completion.

\section{Feedback and Flexibility}

In an effort to understand the environments in which students were trying to learn, students were asked to fill out an anonymous survey indicating if they had unlimited access to the internet, a quiet space to watch lectures, or child or elder care responsibilities. The responses to these questions guided the approach to be as flexible as possible, while still having hard deadlines to encourage students to work on the course steadily throughout the term instead of trying to complete everything at the end. The day that every module was due, an email was sent to students who had not yet turned in their homework or quiz, with a reminder that it was due that night, but they could ask for an extension with no penalties. Less than five students asked for an extension for each module, with a different set of students asking each time. Students 
were not required to give a reason as to why they needed an extension, but those that did often cited events that were disruptive to their well-being and/or learning environment. No student cited internet access as a reason for needing an extension. Overall, being highly flexible and compassionate with deadlines addressed the challenge of ensuring accessibility to all students.

Another anonymous feedback survey was taken halfway through the quarter, where students provided feedback on what aspects of the class were going well, what was challenging, what parts of the courses aided their learning the most, and any suggestions for improvement. From this feedback more structure was provided on how to work effectively in groups remotely and more unstructured group project time was built into the last few weeks of the class. This further addressed the challenge of fostering teamwork within group projects.

\section{REFLECTION}

\section{Course Learning Objectives}

Although the delivery of this course differed from previous iterations, overall the course learning objectives were still met by the updated versions of each course component. The only part of the course learning objectives that was not able to be fully met was the ability to take measurements on living systems, since students could not use the biosensors. If this class were to be taught remote again, a small assignment could be created where students took measurements that did not require biosensors such as heart rate or breathing rate on themselves. Furthermore, to better replace the hands-on components, virtual labs could be created of a selection of biosensors so that students felt better prepared to choose one for their design of an experiment group project.

\section{Lectures}

A flipped classroom approach to undergraduate engineering courses increases engagement with the course content. ${ }^{4}$ Furthermore, providing the lecture material as both written handouts and recorded videos ensured students could learn the material in a manner that worked best for them. ${ }^{8}$ Although all of the information was available in the handouts, the majority of the class watched all the videos for each module. Lastly, breaking the lectures up into 5-10 min clips compared to one long video had multiple positive effects. Students absorb material better when available in shorter clips compared to one long one, ${ }^{3}$ and providing the information in well labeled short clips meant students could easily navigate to the video corresponding to the questions they got wrong on their first quiz attempt. The lectures, homework, and quizzes all complemented each other and helped students meet the course learning objectives \#2 and \#3.

\section{Homework}

Approximately $75 \%$ of the students attended the Zoom lectures where we went over the homework together, and the recorded lectures were watched by over half the students, indicating that even those that came to lectures went back and watched the videos of the homework solutions in order to be able to complete the homework assignments. Students did not ask a lot of questions during the homework sessions while they were in breakout rooms, but they did engage with the homework material and ask questions when they returned to the main room to go over the homework solutions. Although the students were given the answers, the act of completing the homework themselves in order to turn in individual assignment ensured process comprehension. Furthermore, working on the homework in small groups helped build a sense of camaraderie and community within the class. ${ }^{6}$ Perceived rapport with both instructors and students increases student participation. ${ }^{2}$ However, some students provided feedback that they didn't feel challenged by the homework, thus in future iterations of this course the homework might be updated slightly. One option would be to have the students turn in the homework before class, grade based on completion not having the right answers, go over the solutions in class, and then give bonus points for having the correct answers. Another option would be to assign one problem per homework that is not gone over in class but is very similar to the most challenging homework question. That way students can see how the method learned from the discussed problem can be applied to a slightly different problem.

\section{Quizzes}

Quiz structure was based off of Khan academy due to their well-established format and position in academic learning. Not making the quizzes timed allowed students to go through the lecture material at their own pace to find the answers and be mindful of student inclusivity. Allowing students to take the quizzes twice encouraged them to identify what they had gotten wrong and find the correct answer, enhancing comprehension. ${ }^{7}$ The majority of the students took the quizzes twice, improving their scores to over $90 \%$. Cheating was not evaluated as the answers to the quiz questions were in the lecture handouts. This element of 
the course paired with the prerecorded lectures could be retained for future iterations of this course.

\section{Participation}

The participation activities of engaging with Science Twitter, taking handwritten notes, and summarizing one aspect of the course material went very well. Handwritten notes enhance comprehension, ${ }^{5}$ but since the course was being delivered remotely students were not motivated to take such notes. Some students uploaded one short page of notes, while others uploaded ten pages or more of very detailed notes for each module. Lastly, explaining a concept to someone much like you are teaching someone else, reinforces comprehension. ${ }^{1}$ Interestingly, very few students chose to upload a video, with the majority writing a short abstract on a topic instead. Overall, these three participation activities provided as much as an assessment of attendance for participation if not more and could be retained for future iterations of this course.

\section{Group Projects}

From the student's point of view the group projects seemed to be the most difficult aspect of this course. There were some elements that went well, and many elements that were improved upon as the course progressed. Manager meetings went very well, with students preparing thoughtful questions and provided a good sense of where they were at progress wise. Lectures that were devoted to working on the group projects had the highest attendance, whether they were scaffolded or not.

Students provided feedback that working remotely with group members was very challenging, with issues ranging from finding a time to all meet up on Zoom to figuring out how to divide the labor amongst the group members. Many of the students thought they needed to be meeting on Zoom in order to do any work on the projects, instead of assigning tasks to each group member, working on them individually, and then meeting to put them all together into a final complete project. Upon receiving this feedback, much more scaffolding was provided in terms of suggested division of labor and some tips on how to work well in groups remotely. Additionally, the first group project had teams of 5-6 students, but there wasn't enough work for 5-6 students to do so many group members expressed frustration that there was an uneven distribution of labor. For the remaining two projects group size was reduced to 4-5 students, which went much better.
Further efforts to reduce group project frustrations could include monitoring of group dynamics and allowing students to choose their own groups for at least one of the group projects. A survey halfway through each group project could be given to students where they are asked how things are going and what could be improved upon. This would allow the instructor to intervene if there were specific problems arising in one or two groups or broadcast a message to all the groups if they were all experiencing similar issues. As for selecting group members, groups were formed automatically using a tool in Canvas that randomly assigned students to groups. This allowed for grades to be entered once per group instead of individually. However, if the students were empowered to select their own groups inter-group dynamics could potentially be improved.

\section{CONCLUSION}

Overall, despite the challenges of remote delivery, the quarter went well, with students engaging well with the course and all students got high grades across the board on homework assignments, quizzes, participation assignments, and group projects. Many elements of this COVID-19 version of the course will be kept for future years, whether in person or online.

\section{ACKNOWLEDGMENTS}

Special thanks to the three outstanding graders of the Spring 2020 class.

\section{CONFLICT OF INTEREST}

The authors have no competing interests.

\section{REFERENCES}

${ }^{1}$ Fiorella L, Mayer RE. Role of expectations and explanations in learning by teaching. Contemp Educ Psychol. 2014:39(2):75-85.

${ }^{2}$ Frisby BN, Martin MM. Instructor-student and studentstudent rapport in the classroom. Commun Educ. 2010;59(2):146-64.

${ }^{3} \mathrm{H}$ sin WJ, Cigas J. Short videos improve student learning in online education. J Comput Sci Coll. 2013;28(5):253-9.

${ }^{4}$ Mason GS, Shuman TF, Cook KE. Comparing the effectiveness of an inverted classroom to a traditional classroom in an upper-division engineering course. IEEE Trans Educ. 2013;56(4):430-5. 
${ }^{5}$ Mueller PA, Oppenheimer DM. The pen is mightier than the keyboard: advantages of longhand over laptop note taking. Psychol Sci. 2014;25(6):1159-68.

${ }^{6}$ Nieto J, Valery S. Creating a sense of community in the classroom. J Pedagog Plur Pract 2006;3(5):71-85.

${ }^{7}$ Rushton SJ. Teaching and learning mathematics through error analysis. Fields Math Educ J. 2018;3(4):1-2.
${ }^{8}$ Zapalska A, Brozik D. Learning styles and online education. Campus-Wide Inf Syst. 2006;23(5):325-35.

Publisher's Note Springer Nature remains neutral with regard to jurisdictional claims in published maps and institutional affiliations. 